\title{
CLASIFICACIÓN DEL DOMINIO COGNITIVO DE LAS FUNCIONES EJECUTIVAS Y LA MEMORIA EN LA BATERÍA NEUROPSI
}

\author{
CLASSIFICATION OF THE COGNITIVE DOMAIN OF EXECUTIVE FUNCTIONS \\ AND MEMORY IN NEUROPSI BATTERY \\ José Carlos Rivera B. ${ }^{1}$ \\ Universidad Nacional Mayor de San Marcos, Lima, Perú \\ (RECibido 30/01/2013. AcEPTAdo 25/4/2013)
}

\begin{abstract}
RESUMEN
Si las pruebas, instrumentos o baterías psicológicas tienen en común algo que las caracteriza es su validez de constructo, es decir, en este caso ¿cómo se agrupó el conjunto de variables a medir en la Batería NEUROPSI?

Se analizaron comparativamente los elementos conceptuales (variables e indicadores) del NEUROPSI Atención y Memoria 6-85 años, ubicados en dos publicaciones especializadas, y se encontró que la Batería utilizada en el estudio de aplicación fue una variante de la referida en la ficha técnica del instrumento.

Centrados en la validez de constructo de las funciones ejecutivas y la memoria,se discutió el alcance que tiene la inclusión del test de Rey (test de percepción y memoria) en las áreas mencionadas, es decir: tomaron como parte del NEUROPSI, la figura compleja de ReyOsterreith en lo referente a la memoria, y dejaron de lado el de la exploración perceptiva, lo que generó una interrogante sobre la validez de constructo y de concurrencia en la Batería.
\end{abstract}

Palabras clave: batería NEUROPSI, funciones ejecutivas, memoria, validez de constructo, test de rey

\begin{abstract}
If tests, psychological instruments or batteries have in common is something that characterizes its construct validity, that is to say, in this case how the set of variables grouped to measure NEUROPSI Battery?

Comparatively analyzed the conceptual elements (variables and indicators) of Attention and Memory NEUROPSI 6-85 years, located in two journals, and found that the battery used in the implementation study was a variant of that in the data sheet instrument.

1 Profesor Asociado de la Facultad de Psicología, investigador del Instituto de Investigaciones psicológicas de la UNMSM. E-mail: jocarivera@gmail.com
\end{abstract}


Focus on the construct validity of executive functions and memory is discussed on the extent of the inclusion of Rey test on perception and memory in these areas: take as part of NEUROPSI, Rey-Osterreithcomplex figure in regarding memory, leaving aside the perceptive exploration, we generated a question of construct validity and concurrency in the Battery.

Keywords: Battery NEUROPSI, executive functions, memory, construct validity, Rey test

\section{INTRODUCCIÓN}

El objetivo de este trabajo es el análisis de la clasificación de dos categorías conceptuales de la psicología cognitiva, las funciones ejecutivas y la memoria, en la Batería NEUROPSI, Atención y Memoria 6-85 años de los autores Feggy Ostrosky-Solís, Esther Gómez Pérez, Esmeralda Matute, Mónica Rosselli, Alfredo Ardila y David Antonio Pineda (2003).

Un uso de esta batería se encuentra en el estudio de aplicación en Guanajuato (México) de los autores Gabriela Castillo-Parra, Esther Gómez Pérez y Feggy Ostrosky-Solís (2009).

La ficha técnica de la batería se ubica en una compilación sobre neuropsicología infantil en lo concerniente a pruebas neuropsicológicas de Esmeralda Matute, Mónica Rosselli, Yaira Chamorro y Julia Carolina Orozco (2010).

El interés particular en las categorías mencionadas se justifican en el marco de nuestro proyecto de tesis doctoral (Rivera, 2012), cuyas fuentes bibliográficas venimos comprobando son escasas, casi inexistentes por lo que estudios como el de 2009 constituyen, aunque no en su conjunto, antecedentes que debemos considerar en cuanto a la instrumentación para medir las variables del proceso cognitivo relacionados con la praxia constructiva y la memoria espacial.

El método hermeneútico nos permite comparar, luego de la exposición de motivos en el caso de la publicación 2009, los dominios de la clasificación de las variables en el NEUROPSI con las de la publicación 2010.

En la introducción de la primera publicación, se afirma que para afrontar los cambios que ocurren en el aprendizaje formal que se da al ingresar a la escuela "se requiere de la atención y la memoria principalmente (...) son condiciones previas indispensables para el funcionamiento adecuado de otros dominios cognitivos, como es el caso de la funciones ejecutivas" (Castillo-Parra, Gómez y OstroskySolís, 2009, p. 42). Allí mismo, se señala que:

Los estudios referentes al desarrollo de la atención, la memoria y las funciones ejecutivas han demostrado que uno de los principales factores que influyen sobre estos tres procesos es la edad (...) Otra variable que influye sobre los procesos cognitivos, y que puede ser todavía más significativa que la edad, es la escolaridad (...) Gómez Pérez y Ostrosky-Solís utilizaron la batería neuropsicológica NEUROPSI, Atención y Memoria (Ostrosky et al., 2003) para evaluar los efectos de la edad y escolaridad sobre la atención, funciones ejecutivas y memoria en un grupo de niños y adolescentes entre 6 
y 15 años y un grupo de adultos entre 15 y 85 años; estos últimos divididos de acuerdo a sus años de escolaridad (...) Estas autoras concluyeron que durante la niñez, la atención selectiva y sostenida, la memoria de trabajo y las funciones ejecutivas tienen un rápido desarrollo, que comienza desde edades tempranas y continúan estables hasta la adolescencia, y que la escolaridad juega un papel importante para el perfeccionamiento de estos procesos cognitivos (Castillo-Parra et al., 2009, pp. 43-44).

Luego, las autoras proponen que la investigación de la atención, las funciones ejecutivas y la memoria no pueden considerarse como constructos independientes. En este sentido se plantearon como objetivo "evaluar los efectos de la capacidad de atención, funciones ejecutivas y memoria sobre el nivel de rendimiento académico alto, medio y bajo en niños de primaria" (Op. cit., p. 44).

Lo que no explicitan estas autoras es cuál es la naturaleza técnica del constructo que unifica la atención, las funciones ejecutivas y la memoria.Supuestos que deben ser aclarados en el debate, comunicación científica y probablemente en futuras investigaciones como las propuestas en las conclusiones de este análisis.

\section{CLASIFICACIÓN DE LAS FUNCIONES COGNITIVAS EN EL NEUROPSI ATENCIÓN Y MEMORIA}

En relación al instrumento de evaluación se encuentran diferencias en la clasificación de los procesos cognitivos de acuerdo al Manual del NEUROPSI Atención y Memoria (Matute, Rosselli, Chamorro y Orozco, 2010) y, la del estudio de aplicación (Castillo-Parra, Gómez y Ostrosky-Solís, 2009). Teniendo en cuenta que en ambas publicaciones participan algunas de las coautoras del Neuropsi (Matute y Rosselli, en la primera, y Gómez y Ostrosky-Solís, en la segunda) consideramos necesario señalar esas diferencias, más aún porque el instrumento cuenta con datos normativos obtenidos de un total de 950 individuos normales mexicanos entre 6 y 85 años de edad.

Según las autoras de la publicación del 2010 el NEUROPSI:

Permite evaluar en detalle tipos de atención entre los que se encuentran la atención selectiva, sostenida y el control atencional; así como tipos y etapas de memoria incluyendo memoria de trabajo, y memoria a corto y largo plazo para material verbal y visoespacial. El NEUROPSI, Atención y Memoria evalúa las siguientes áreas:

I. Orientación

II. Atención y concentración

a) Deficiencias en el nivel de conciencia o estado de activación (entrevista).

b) Atención selectiva (detección visual, retención de dígitos en progresión y cubos en progresión).

c) Atención sostenida (detección de dígitos y series sucesivas).

d) Control atencional (fluidez verbal semántica y fonológica, fluidez no verbal, funciones motoras y la prueba de Stroop). 
III. Memoria (codificación y evocación de material verbal [palabras aisladas, pares de palabras y párrafos] y de material visual [figura semicompleja, figura compleja de Rey-Osterreith y caras], dígitos en regresión y cubos en regresión). (Matute et al., 2010, pp. 133).

Veamos ahora, la clasificación que se hace de la misma batería NEUROPSI Atención y Memoria en la publicación del 2009:

La batería examina dos principales áreas a través de un conjunto de pruebas. $\mathrm{El}$ área de atención y funciones ejecutivas que incluye orientación (tiempo, persona y espacio), atención y concentración (dígitos y cubos en progresión, detección de dígitos, rastreo visual y series sucesivas), memoria de trabajo (dígitos y cubos en regresión), funciones ejecutivas (formación de categorías, fluidez semántica, fonológica y no verbal, flexibilidad, inhibición y tareas de programación motora), y el área de memoria que se evalúa a través de la etapa de codificación (memoria verbal y no verbal, memoria lógica, pares asociados y caras) y la etapa de evocación (memoria verbal espontánea, por claves y reconocimiento, memoria no verbal, memoria lógica, pares asociados y nombre e identidad de caras). Esta batería cuenta con datos normalizados para población mexicana de acuerdo a un rango de edad de 6 a 85 años (Castillo-Parra et al., 2009, p. 46).

Seguidamente comparamos las áreas que evalúa el NEUROPSI según Matute et al. (2010) y la que acabamos de exponer, encontrando que varios ítems no coinciden como se muestra en la Figura 1:

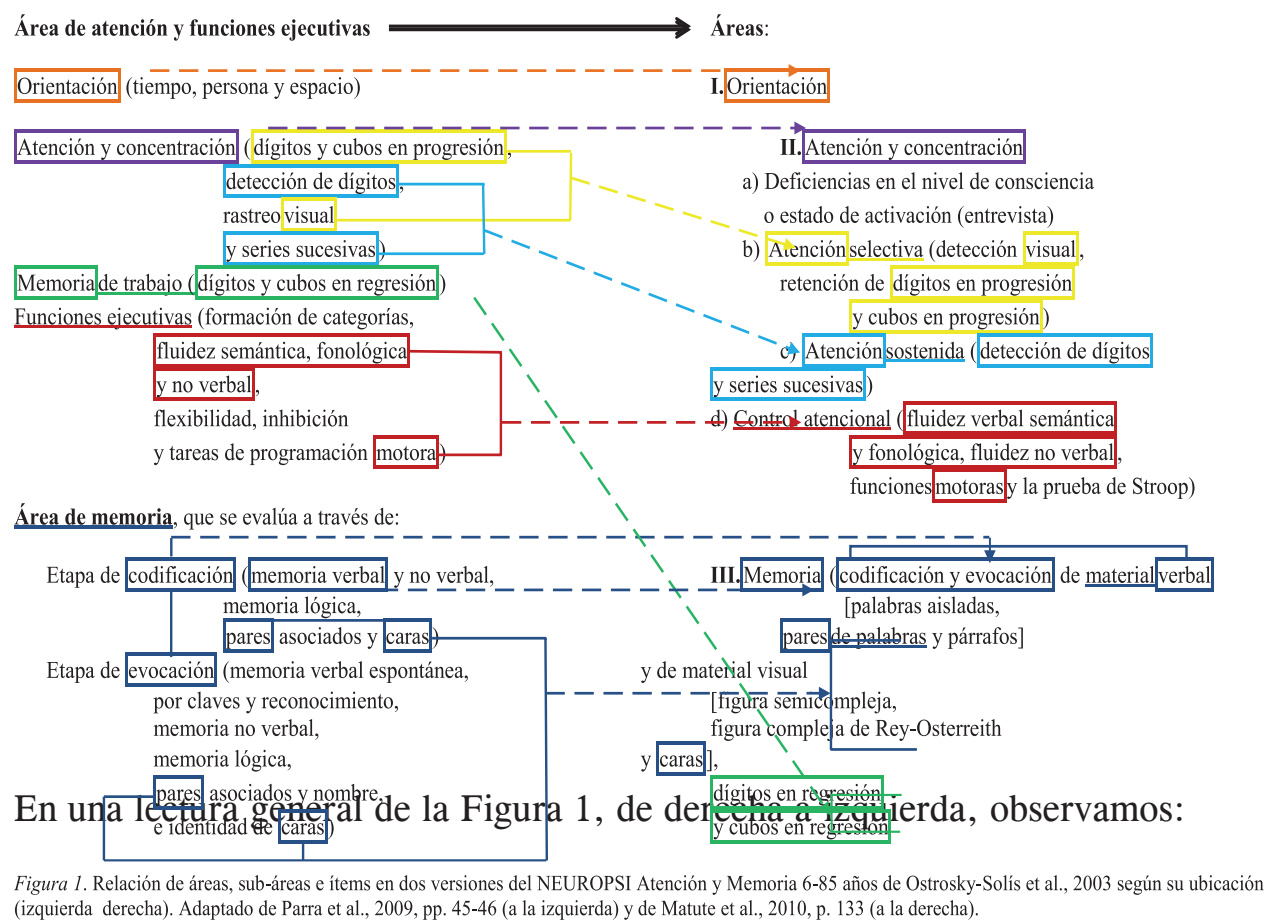


- Reagrupación de las áreas I Orientación y II Atención y concentración en una denominada Área de atención y funciones ejecutivas.

- Las sub-áreas II b) Atención selectiva y II c) Atención sostenida se agrupan en una sub-área del mismo nombre del Área II Atención y concentración.

- La sub-área II d) Control atencional se agrupan en una sub-área denominada Funciones ejecutivas.

- El área III Memoria se mantiene en su mayor parte, salvo por los ítems dígitos y cubos en regresión que se agrupan en una sub-área Memoria de trabajo.

Constatamos entonces que para la aplicación referida en la publicación 2009 se redimensionaron las áreas respecto a las señaladas en la ficha técnica publicada en el 2010.

\section{FUNCIONES EJECUTIVAS Y MEMORIA EN EL NEUROPSI ATENCIÓN Y MEMORIA}

Los autores del NEUROPSI (Ostrosky-Solís et al., 2003. Citado en Matute et al., 2010, p. 133) incluyen la prueba de Rey-Osterreith en el área III Memoria. Allí mismo, a la prueba de Rey l--a clasifican como un test de "Habilidades Construccionales y Memoria" (Op. cit., p. 134).

André Rey (1997), quien denomina a su prueba como "Test de Copia y Reproducción de Memoria de Figuras Geométricas Complejas", tiene como objetivo conocer la actividad perceptiva, mediante el proceso de copiado de un diseño geométrico complejo, el cual es reproducido en una segunda fase para conocer el grado y fidelidad de la memoria visual.

Rey postula que el proceso de exploración perceptiva influye en la reproducción mnémica. Por lo tanto un defecto en la exploración perceptiva conlleva una reproducción mnémica defectuosa, pero no atribuible a la memoria necesariamente. También, respecto al alcance de su instrumento, el autor señala:

Es necesario precisar que la percepción estudiada aquí es una percepción visual, directriz de un proceso de copia, siendo esta percepción visual la que centra la actividad. La percepción visual podría presentar otras características si la examinásemos orientándola hacia otros fines y asociada a otras actividades. Esta reserva invita a la prudencia en la interpretación de algunos resultados de aspecto patológico (Rey, 1997, p. 8).

En esta línea de análisis se observa que los autores del Neuropsi toman la prueba de Rey-Osterreith como un indicador de memoria (Figura 1, derecha) sin considerar que para Rey el constructo del test se basa en el proceso de percepción y memoria visual.

Así mismo, según la clasificación del Neuropsi en Castillo-Parra et al. (2009) la sub-área denominada Funciones ejecutivas (Figura 1, izquierda) solo lo sería en lo que respecta a la programación y funciones motoras, ligadas a la inteligencia ejecutiva (Borelli y Oléron, 1964/2010)las cuales, por lo tanto, también pueden 
explorarse mediante el proceso de copia en la prueba de Rey-Osterreith ya que este aspecto, además, se relaciona con la organización práxica de la conducta, la cual durante el desarrollo cognitivo se va complejizando por la adquisición de nociones espaciales y estas son cualitativamente diferentes entre niños de 8 y 12 años, tal como lo ha demostrado Piaget al afirmar que la noción de espacio es dominantemente proyectiva a los 8 años y euclideana a los 12 años (Droz y Rahmy, 1984, pp. 169-171).

\section{DISCUSIÓN}

Por lo expuesto consideramos que sería interesante relacionar el test NEUROPSI con tests de praxia constructiva, nociones espaciales, inteligencia ejecutiva y memoria espacial, estableciendo como criterio de medida la discriminación genética para edades de 6 a 14 años en función del rendimiento académico alto, medio y bajo.

De esta manera, cubriendo grados y edades intermedias podríamos obtener indicadores que nos den información sobre los cambios evolutivos en las funciones ejecutivas y memoria, ya que la desorganización visoespacial conlleva algunas veces un síntoma de apraxia constructiva (Portellano, 2005, pp. 266-267).

De otro lado, la desorganización visoespacial se puede solapar con la ausencia de nociones espaciales, los cuales no se han estudiado en su evolución psicogenética, ya que el factor educación formal tendría una mayor influencia dado su carácter epigenético, lo que no ocurre necesariamente con la organización visoespacial relacionado con la maduración del sistema nervioso.

Considerando que no se refiere la aplicación del aspecto perceptivo de la prueba de Rey-Osterreith, según la descripción del NEUROPSI (2010), se genera una interrogante respecto a la validez de constructo, al tener en cuenta solo la parte correspondiente a memoria visual.

De otro lado, la programación motora a partir de que el niño realiza representaciones mediante el dibujo ya es de carácter representacional, y la evolución de las nociones espaciales influyen en la orientación espacial y otros aprendizajes perceptuales.

\section{CONCLUSIONES}

Dicho esto, para mejorar la validez de constructo y enriquecer la validez concurrente del test NEUROPSI, consideramos interesante realizar las siguientes propuestas a manera de conclusión:

1) Necesidad de correlacionar el área de funciones ejecutivas y memoria del NEUROPSI con el test de Rey-Osterreith de percepción y memoria visual, en relación a las clasificaciones de las versiones descritas en la Figura 1. 
2) Necesidad de correlacionar los niveles de las nociones representativas del espacio, topológicas, proyectivas y euclideanas, propuestas por Jean Piaget, con las obtenidas de acuerdo a la conclusión primera.

\section{REFERENCIAS BIBLIOGRÁFICAS}

Borelli, M. y Oléron, P. (1964/2010). Manual de la Escala Ejecutiva Borelli-Oléron. En Rivera, J. C. Estudio de la Imagen Reproductora Estática y Cinética en Sujetos con Retardo Mental Leve y Moderado del Programa de Educación Especial. (Tesis de Maestría, en prensa Cybertesis). UNMSM. Lima, 2011.

Castillo-Parra, G., Gómez Pérez, E. y Ostrosky-Solís, F. (2009). Relación entre las Funciones Cognitivas y el Nivel de Rendimiento Académico en Niños. Revista Neuropsicología, Neuropsiquiatría y Neurociencias, Vol. 9, N. ${ }^{\circ}$ 1, pp. 41-54. Recuperado de: http://neurociencias.udea.edu.co/revista/PDF/REVNEURO_vol9_num1_8.pdf

Droz, R. y Rahmy, M. (1984). El desarrollo de las funciones cognoscitivas. En Cómo Leer a Piaget. (Trad. A. Romero). Cap. III, Segunda Parte, Acáp. 3 (pp. 97-188). México: Fondo de Cultura Económica. (Original en francés, 1978).

Matute, E., Rosselli, M., Chamorro, Y. y Orozco, J. (2010). Pruebas neuropsicológicas infantiles en español. En Rosselli, M., Matute, E. y Ardila, A. Neuropsicología del desarrollo infantil. Cap. 5 (pp. 119-136). México: El Manual Moderno.

Portellano, J. A. (2005). Las apraxias. En Introducción a la neuropsicología. Cap. 9 (pp. 251-269). Madrid: McGraw-Hill.

Rey, A. (1997). Test de copia y de reproducción de figuras geométricas complejas. Madrid: TEA (Original en francés).

Rivera, J. C. (2012). Praxia Constructiva y memoria espacial en el retardo mental. (Proyecto de tesis doctoral). Facultad de Medicina. UNMSM. 